\title{
Abortion-inducing drug alarms the right-to-life lobby
}

\section{Washington}

A NEW steroidal compound that may be used for treating cancer, glaucoma and even AIDS (acquired immune deficiency syndrome) is nonetheless at the centre of a political storm because of its most promis. ing property: the ability to terminate early pregnancy with a single, $600-\mathrm{mg}$ dose. The drug, produced by the French pharmaceutical company Roussel-UCLAF, is most commonly referred to by its original code name, RU 486. But opponents of the testing of RU 486 have called it the 'death pill'; they are asking that it should never be made available in the United States.

RU 486 is an antiprogestin that is thought to compete with progesterone at the receptor site. The antihormone-receptor complex cannot trigger the actions normally provoked by the hormone. During pregnancy, the antiprogesterone increases contractility of the uterine muscles, an effect enhanced by prostaglandins. Administration of RU 486 will induce menses in woman who are not pregnant, and abortions - in most cases - in women who are.

RU 486 is also an antiglucocorticosteroid, which makes it useful in treating certain forms of Cushing's syndrome, a disease characterized by increased adrenocortical activity. Similarly, RU 486 may be used to treat other conditions related to excess corticosteroid production, such as lymphomas and glaucoma. By blocking cortisol activity at the receptor level, RU 486 can also interrupt the normal negative feedback control mechanism, stimulating the hypothalamic-pituitaryadrenal axis. This activation can enhance the effect of anticancer drugs, and alter the immune response, potentially relevant to treating AIDS patients.

Approximately 1,500 women around the world have now been given RU 486 to terminate pregnancy. The World Health Organization is conducting a multicentre trial in eight countries (China, India, Hungary, Sweden, Singapore, Vietnam, Italy and Scotland) using a combination of RU 486 and prostaglandins in tandem to induce abortions. The Population Council has also organized studies of RU 486's effectiveness as an abortion inducer both in the United States and overseas. Roussel-UCLAF has conducted its own clinical trials in France. In one such trial, recently reported, RU 486 successfully terminated pregnancy in 85 of 100 women treated within 10 days of their first missed period.

Supporters of the right-to-life movement in the United States are alarmed by the implications of RU 486, which they fear will make abortions easier to arrange, and at the same time diminish a woman's sense that she is taking the life of her unborn child. A group of 6 conservative senators wrote to Secretary of Health and Human Services Otis Bowen last February, asking him to confirm that federal funds were not being used to investigate RU 486 as a potential agent for abortion.

In April, Bowen responded with a list of all projects using RU 486 sponsored by the National Institutes of Health (NIH). NIH have been scrupulously careful about not using their facilities in any way that might be considered related to abortion. Federal law prohibits federal money from being spent on abortion research. Tests on human subjects are all carried out before implantation of the fertilized egg.

Etienne-Emile Baulieu of the Université Paris-Sud, who has developed RU
486 , would prefer that the drug should be called a contragestive agent rather than an abortifacient, on the grounds that it interferes with the establishment of pregnancy. But he admits that the political antecedents of the drug may impede its introduction in the United States, where liability issues could also price the drug out of a potentially enormous market.

There are other than social problems for RU 486. The drug has a relatively long half-life - 20 hours - allowing it to remain in the body longer than would optimally be desired. Heavy bleeding follows treatment in some women, which means that taking the drug without medical supervision could prove dangerous.

Despite these problems, other companies are interested in the potential of RU 486. Schering AG in West Germany has developed a similar compound now in clinical trials. And Roussel-UCLAF plans to submit licence applications for RU 486 this summer in France and Sweden.

Joseph Palca

\section{New Australian law on embryos still confuses researchers}

\section{Sydney}

What is an embryo? The answer could provide a solution to some of the issues raised by the Infertility Medical Procedures Act which came into effect last $\mathrm{Au}$ gust in the state of Victoria, Australia. The threat of four-year gaol terms contained in the act brought in vitro fertilization (IVF) research in Victoria to a complete halt and prompted Dr Alan Trounson, the leader of Monash University's world-renowned IVF research team, to issue an ultimatum that he and his team would go overseas within six months if they were not allowed to continue research. Three months have now passed (see Nature 232, 748; 1986).

The legislation forbids the destruction of embryos but it never actually defines an embryo and at what point a fertilized egg becomes an embryo, or even what fertilization is. According to Professor Carl Wood, chairman of the department of obstetrics and gynaecology at Monash University and head clinician of the Monash/ Queen Victoria Hospital IVF programme, all efforts towards a resolution of the researchers' crisis have been stalled by the notion that an embryo comes into being the moment the sperm head passes into the shell of an egg. Although this is an essential step, there are others that might more logically mark the beginning of an embryo's existence, such as 20 hours after fertilization when the male and female genetic material fuse together or 14 days which mark the first appearance of cells that will have a lineage to the fetus and eventually to the new human. Before the 14th day, all the cells that grow from the fertilized egg go to make up the support systems for the fetus such as the fetal sac and the placenta.

The Standing Review and Advisory Committee on Infertility chaired by Professor Louis Waller has power to adjudicate on the interpretation of the legislation. A request from the Monash scientists for clarification of terms such as embryo and fertilization used in the legislation, as well as what constitutes an abnormal embryo and when such can be discarded, is now before the Waller committee. Adoption of a 20-hour limit for embryos should clear the way for approval of two proposed experiments before the Waller committee. This would be sufficient to ensure that the Monash team stays.

One experiment promises the development of a new method for the treatment of male infertility, fertilization by the microinjection of single sperm, and the other is on the gamete freezing method which involves the freezing and thawing of egg cells. Both experiments involve the fertilization of eggs without implantation - an illegal act while fertilized eggs are classified as embryos.

The Monash team would like to see the adoption of the term 'pre-embryo' for the initial 14-day period as used in Britain; this might eventually lead to a broader range of permissible experimentation.

There is still no indication from the Waller Committee on when it will make its rulings. Trounson sees some irony in the situation. If such a law had been in force six years ago, thousands of families would still be childless. 